\title{
Preliminary biocompatibility investigation of magnetic albumin nanosphere designed as a potential versatile drug delivery system
}

This article was published in the following Dove Press journal:

International Journal of Nanomedicine

17 August 201 I

Number of times this article has been viewed

\author{
Luciana Estevanato' \\ Débora Cintra' \\ Nayara Baldini' \\ Flávia Portilho' \\ Luzirlane Barbosa' \\ Olímpia Martins ${ }^{2}$ \\ Bruno Lacava ${ }^{3}$ \\ Ana Luisa Miranda-Vilela' \\ Antônio Cláudio Tedesco² \\ Sônia Báo' \\ Paulo C Morais ${ }^{4}$ \\ Zulmira GM Lacava' \\ 'Instituto de Ciências Biológicas, \\ Universidade de Brasília, \\ 2Departamento de Química, \\ Laboratório de Fotobiologia \\ e Fotomedicina, Faculdade de Filosofia, \\ Ciências e Letras de Ribeirão \\ Preto, Universidade de São Paulo, \\ Ribeirão Preto, ${ }^{3}$ Instituto de Química, \\ Universidade de Brasília, Brasília, \\ ${ }^{4}$ Instituto de Física, Universidade \\ de Brasília, Brasília, Brazil
}

Correspondence: Zulmira GM Lacava

CNANO, Instituto de Ciências

Biológicas, Universidade de Brasília,

70910-900, Brasília-DF, Brazil

$\mathrm{Tel} / \mathrm{Fax}+556131073087$

Email zulmira@unb.br
Background: The magnetic albumin nanosphere (MAN), encapsulating maghemite nanoparticles, was designed as a magnetic drug delivery system (MDDS) able to perform a variety of biomedical applications. It is noteworthy that MAN was efficient in treating Ehrlich's tumors by the magnetohyperthermia procedure.

Methods and materials: In this study, several nanotoxicity tests were systematically carried out in mice from 30 minutes until 30 days after MAN injection to investigate their biocompatibility status. Cytometry analysis, viability tests, micronucleus assay, and histological analysis were performed.

Results: Cytometry analysis and viability tests revealed MAN promotes only slight and temporary alterations in the frequency of both leukocyte populations and viable peritoneal cells, respectively. Micronucleus assay showed absolutely no genotoxicity or cytotoxicity effects and histological analysis showed no alterations or even nanoparticle clusters in several investigated organs but, interestingly, revealed the presence of MAN clusters in the central nervous system (CNS).

Conclusion: The results showed that MAN has desirable in vivo biocompatibility, presenting potential for use as a MDDS, especially in CNS disease therapy.

Keywords: nanotoxicity, nanoparticle, genotoxicity, cytotoxicity, brain

\section{Introduction}

The last four decades have been marked by huge interest in the development of drug delivery systems (DDS), with special emphasis on the design and fabrication of DDS for cancer treatment. In general, DDS are engineered to minimize drug degradation upon administration, prevent undesirable side effects, and sustain and/or increase the drug's bioavailability in the targeted area. ${ }^{1-3}$

More recently, a new generation of DDS, which is in association with magnetic nanoparticles (MNPs), has become particularly interesting. A MNP-based DDS (MDDS) may not only present enhanced structural stability, tissue absorption, and targeting while reducing side effects, ${ }^{2,4}$ but also reveal specific properties of MNPs, including the potential to carry out hyperthermia ${ }^{5,6}$ and thermoablation of tumor cells, magnetic resonance imaging contrast enhancement, gene therapy, magnetic separation, and even offer the possibility of being site-guided or localized in a specific target tissue by external gradient of magnetic fields. ${ }^{7-9}$

Magnetohyperthermia is a particularly interesting application of MNPs: after binding to cancer cells, MNPs can be submitted to an external alternating magnetic field, promoting a mild increase in local temperature. This increase can cause the preferential destruction of cancer cells, with minor effects to neighboring normal cells, ${ }^{1}$ because 
tumor cells are less resistant to the sudden rise in temperature than the surrounding normal cells. Magnetohyperthermia is currently used to improve cancer treatments when used in combination with radiotherapy or chemotherapy. ${ }^{10}$

To be used as MDDS, as for instance in magnetohyperthermia protocols, the magnetic phase requires high magnetic susceptibility, as can be found in ionic magnetic fluid (MF) samples. However, the drawback to this approach is that ionic MF samples are not biocompatible materials. Indeed, appropriate surface functionalization of the suspended MNPs in MF samples is important to avoid particle clustering while promoting higher biocompatibility and extended circulation time. ${ }^{3,11}$ Protein polymeric-based templates, such as albumin, have been developed to produce biocompatible nanocomposites for the engineering of DDS. ${ }^{12,13}$ An illustrative example is Abraxane ${ }^{\circledR}$ (Celgene Corp, Summit, NJ), an albumin-stabilized nanoparticulated formulation of paclitaxel, presenting higher antitumoral activity in patients with metastatic breast cancer while inducing minor side effects compared to the free drug. ${ }^{14}$

Taking all of these aspects into account, a recently developed MDDS is introduced and biologically tested in this study. To produce the new MDDS, maghemite nanoparticles, suspended as a stable ionic MF sample, were subsequently encapsulated within albumin-based nanospheres, from now on labeled magnetic albumin nanospheres (MAN). Bearing in mind that in vivo tests involving nanoparticulated systems represent a key aspect of current investigations in nanotoxicology, this study aims to evaluate the as-produced MAN using female Swiss mice as the animal model. The performance of MAN as a MDDS depends on its biocompatibility profile and biodistribution parameters; these in turn depend to a great extent upon the interaction between the nanoparticulated system and cells. For these reasons, MAN-cell interaction was investigated in this study. In particular, MAN was evaluated with regard to its biocompatibility and biodistribution. The findings lead to the conclusion that MAN has good biocompatibility, presents enormous potential for use as a MDDS, and is able to be used in a variety of applications, including magnetohyperthermia and photodynamic therapy, as reported elsewhere. .,15 $^{6}$

\section{Materials and methods}

\section{Chemicals}

Eosin methylene blue (Wright and Giemsa formulations), trypan blue and cyclophosphamide, and fetal bovine serum (FBS) were purchased from Vetec Fine Chemistry Ltd (Rio de Janeiro, Brazil), Sigma-Aldrich Co (São Paulo, Brazil), and Sorali Biotechnology (São Paulo, Brazil), respectively.
Davidson liquid was prepared by mixing $40 \mathrm{~mL}$ of glycerin, $40 \mathrm{~mL}$ of $40 \%$ formaldehyde, $120 \mathrm{~mL}$ of $95 \%$ ethanol, and $120 \mathrm{~mL}$ of distilled water. All other chemicals were of analytical grade and used without further purification.

\section{Magnetic nanospheres}

Maghemite $\left(\gamma-\mathrm{Fe}_{2} \mathrm{O}_{3}\right)$ nanoparticles (NPs), suspended as low-pH ionic magnetic fluid containing $2.3 \times 10^{17}$ particles $/ \mathrm{mL},{ }^{16}$ were dispersed in aqueous medium containing bovine serum albumin (BSA) in order to produce the MAN sample, following the protocol already described in the literature. ${ }^{17} \mathrm{MAN}$ was prepared following the heat denaturation method at $100^{\circ} \mathrm{C}$ of an aqueous solution containing bovine serum albumin (BSA) under mechanical stirring at 13,000 rpm for 20 minutes. Then an aliquot of the ionic magnetic fluid sample containing maghemite nanoparticles was added to the BSA aqueous solution. The synthesis of the ionic magnetic fluid was performed following the methodology described by Morais et al. ${ }^{18}$ Shortly afterwards, ionic magnetic fluid with maghemite NPs $\left(\gamma-\mathrm{Fe}_{2} \mathrm{O}_{3}\right)$ was synthesized by the coprecipitation method of $\mathrm{Fe}$ (II) and $\mathrm{Fe}$ (III) salts with ammonium hydroxide. After removing the aqueous solution, nitric acid was added to the precipitate until $\mathrm{pH} 3$ was reached.

Transmission electron microscopy showed that MAN and maghemite NPs presented an average diameter of $73.0 \pm 3.0 \mathrm{~nm}$ and $8.9 \pm 0.1 \mathrm{~nm}$, respectively, by analysis micrographs (Figure 1). The average diameter of MAN and maghemite NPs was obtained by computer analysis, using

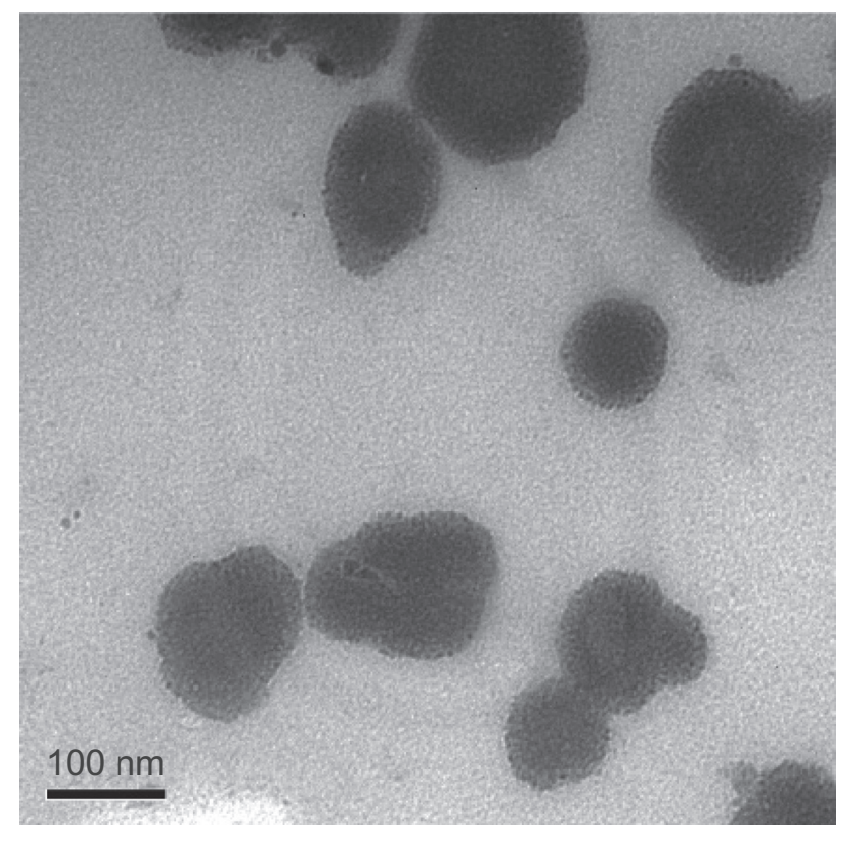

Figure I Transmission electron photomicrography of magnetic albumin nanospheres. 
the program Image-Pro Plus 5.1 (MediaCybernetics, Inc, Bethesda, MD). The distribution of particles was achieved using the best fit log normal. The maghemite content in the MAN sample was about $25 \%$ in mass and about $7 \%$ in volume fraction. At this maghemite particle content the particle-particle interaction is quite effective and the magnetocrystalline anisotropy energy is around $1.119 \mathrm{eV}^{6}$

\section{Animals}

Female Swiss mice, aged 8-10 weeks and weighing 18-22 g, obtained from the animal facility of the University of São Paulo, were housed in plastic cages ( 3 or 6/cage) at room temperature $\left(20^{\circ} \mathrm{C} \pm 2{ }^{\circ} \mathrm{C}\right)$, kept under a 12-hour light/dark cycle with lights on at 6 am and free access to food and water.

\section{Experimental design}

To perform the in vivo application, lyophilized MAN was dispersed in FBS to obtain an aqueous suspension containing $5 \mathrm{mg}$ MAN/mL $\left(1.23 \times 10^{14} \mathrm{MAN} / \mathrm{mL}\right)$. Mice were randomly allocated in groups that were intraperitoneally treated with a bolus dose of (1) $100 \mu \mathrm{L}$ of MAN suspension $(n=6)$, (2) $100 \mu \mathrm{L}$ of FBS $(n=3)$, or (3) received no treatment $(n=6)$. Groups 1 and 3 were used as control.

Biocompatibility evaluation of MAN was performed by (a) total and differential leukocyte counts; (b) viability assay of peritoneal leukocytes; (c) genotoxicity and cytotoxicity studies in bone marrow; and (d) histological analysis of the brain, liver, lungs, spleen, pancreas, heart, and kidneys. Tests a, b, and d were performed at 30 minutes, 6, 12, 24, and 48 hours, and 7, 15, and 30 days after treatment, whereas test c was carried out at 24 and 48 hours, 7, 15, and 30 days after MAN or FBS administration. Histological analyses were also used to evaluate the MAN biodistribution profile. For the genotoxicity and cytotoxicity studies, a positive control group $(n=6)$ with intraperitoneally administered cyclophosphamide was included.

The present animal research was approved by the Animal Ethics Committee of the Institute of Biological Sciences, University of Brasília, Brazil.

\section{Total and differential leukocyte counts}

An aliquot $(20 \mu \mathrm{L})$ of blood, collected through cardiac puncture, was homogenized with $380 \mu \mathrm{L}$ of Turk's solution for 2 minutes, and total white blood cells were counted visually in a Neubauer chamber using a light microscope (Axiophot; Carl Zeiss AG, Oberkochen, Germany). For the differential leukocyte count, ${ }^{19}$ blood smears or peritoneal cells imprinted in glass slides were fixed in methanol for
5 minutes, stained with Wright-Giemsa dye, and observed under light microscope $(n=500$ cells $)$.

\section{Cell viability assay}

Immediately after animal death, viable cells were identified by exclusion of trypan blue dye, which penetrates into the cells with reduced vitality. For the assay, $10 \mathrm{~mL}$ of phosphate buffered saline (PBS), $4^{\circ} \mathrm{C}, \mathrm{pH} 7.2$, were injected into the peritoneal cavity. Subsequently, $8 \mathrm{~mL}$ of the peritoneal liquid were collected and centrifuged in a Dynac ${ }^{\mathrm{TM}}$ centrifuge (Clay Adams ${ }^{\mathrm{TM}}$, NJ) at $160 \mathrm{~g}$ for 5 minutes. The pellet was resuspended in cold PBS and diluted (1:5) with trypan blue solution $(0.4 \%)$. Viable cells (presenting bright yellow color) and dead cells (stained in blue color) were counted in a Neubauer chamber using a light microscope.

\section{Genotoxicity and cytotoxicity evaluations} Micronucleus (MN) assay and the frequency of polychromatic erythrocytes (\%) were used to study MAN genotoxicity and cytotoxicity, respectively. The assay was performed with bone marrow erythrocytes. The femur bone marrow was collected with $1 \mathrm{~mL}$ of FBS and the cells centrifuged (160 $\mathrm{g}$ for 5 minutes). Supernatant was discarded and $50 \mu \mathrm{L}$ of FBS were added to the pellet. Subsequently, $10 \mu \mathrm{L}$ of suspension were smeared on glass slides, fixed by methanol for 5 minutes, and stained with Giemsa dye. Under the light microscope, 4000 erythrocytes were counted - 2000 polychromatic erythrocytes (PCE) and 2000 normochromatic erythrocytes (NCE) - while registering the number of MN found in each cell type. For the evaluation of the inducedMAN cytotoxicity, the number of PCE and NCE was also registered when either of these two cell populations reached the value of 2000. As a positive control group, six mice were intraperitoneally treated with cyclophosphamide at $4 \mathrm{mg} / \mathrm{kg}$ and the bone marrow red cells analyzed after 24 hours. ${ }^{19}$

\section{Histological analyses}

Histological analyses were performed to assess MANinduced morphological effects and biodistribution aspects. ${ }^{20}$ Brain, liver, lungs, spleen, pancreas, heart, and kidneys were surgically removed. Portions of these organs were fixed in Davidson liquid at $4^{\circ} \mathrm{C}$ overnight, dehydrated by alcohol, followed by the diaphanization process using xylene, and subsequently embedded in paraffin blocks. Tissue sections of $5 \mu \mathrm{m}$ thickness were stained with hematoxylin-and eosin or by Perls' reaction. The tissue morphology was observed under a light microscope and the photomicrographies taken by an AxioCam MRc (Carl Zeiss AG). 


\section{Statistical analysis}

Statistical analysis was carried out using Minitab ${ }^{\circledR} 15$ (Minitab Inc, State College, PA) software. Data were expressed as mean \pm standard deviation (SD) and statistical significance was accepted at a level of $P<0.05$. Normal distribution and homogeneity of data variances were verified by Shapiro-Wilk and Levene tests, respectively. Differences between the groups were investigated through analysis of variance (ANOVA), and Tukey's post-hoc test was chosen to carry out 2-to-2 comparisons between the treatments.

\section{Results}

\section{Total and differential leukocyte counts}

No significant differences were observed between control groups (Groups 2 and 3). Thus, only data providing comparisons between Group 1 (MAN-treated) and control Group 3 are shown in Figure 2. With regard to the blood samples, our data indicate that the MAN treatment causes only a small number of significant decreases in the eosinophil population after 6 hours $(P=0.029)$, in neutrophils after 24 hours
$(P=0.035)$, and in total number of both leukocytes $(P=0.042)$ and lymphocytes $(P=0.031)$ after 7 days (see Figure $2 \mathrm{~A}$ ). Some of the peritoneal cavity white cell populations also presented significant but temporary increases in neutrophil and eosinophil populations after MAN application, mainly up to 7 days after injection (see Figure 2B).

\section{Cell viability assay}

A significant decrease was observed in the percentage of viable cells at 24 hours (57\%) and 15 days (73\%) after MAN administration (see Figure 3), compared to the control group (87\%). No significant differences were observed between the control groups in the time window of our experiments.

\section{Genotoxicity and cytotoxicity studies}

The analyses indicated that MAN induced no significant increase in micronucleus frequency in either polychromatic or normochromatic bone marrow erythrocytes (Table 1). Whereas the micronucleus frequency was never higher than $4.33 \pm 2.94(P=0.261)$ after MAN treatment, the
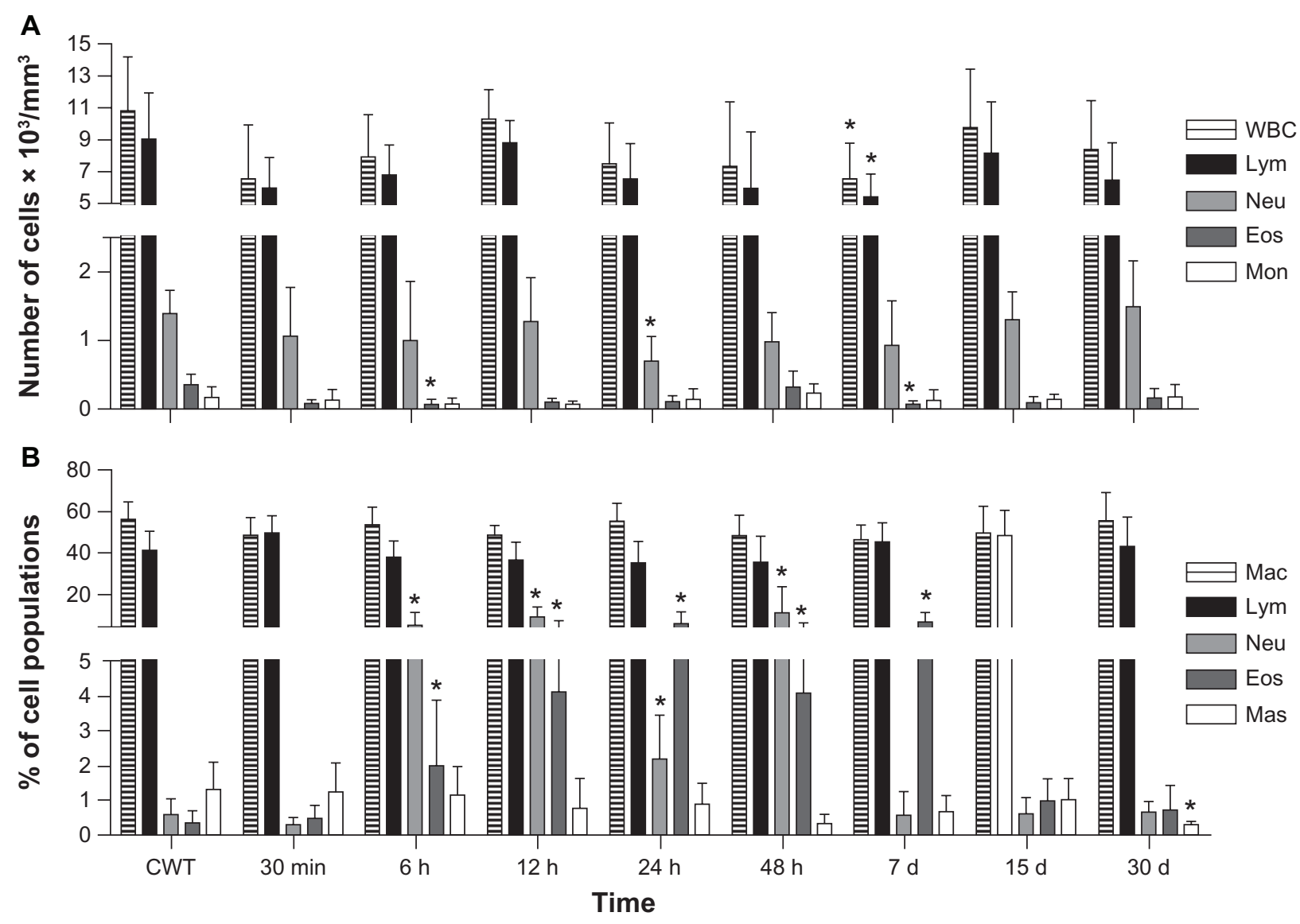

Figure 2 Effects of MAN administration on blood $(\mathbf{A})$ and peritoneal $(\mathbf{B})$ leukocytes as a function of time.

Notes: The data correspond to the means and to the standard deviation; *significant differences between CWT and MAN treatment by Tukey's post-hoc test: $P<0.05$. Abbreviations: CWT, control group without treatment; WBC, global white blood cells; Lym, lymphocytes; Neu, neutrophils; Eos, eosinophils; Mon, monocytes; Mac, macrophages; Mas, mastocytes; MAN, magnetic albumin nanosphere. 


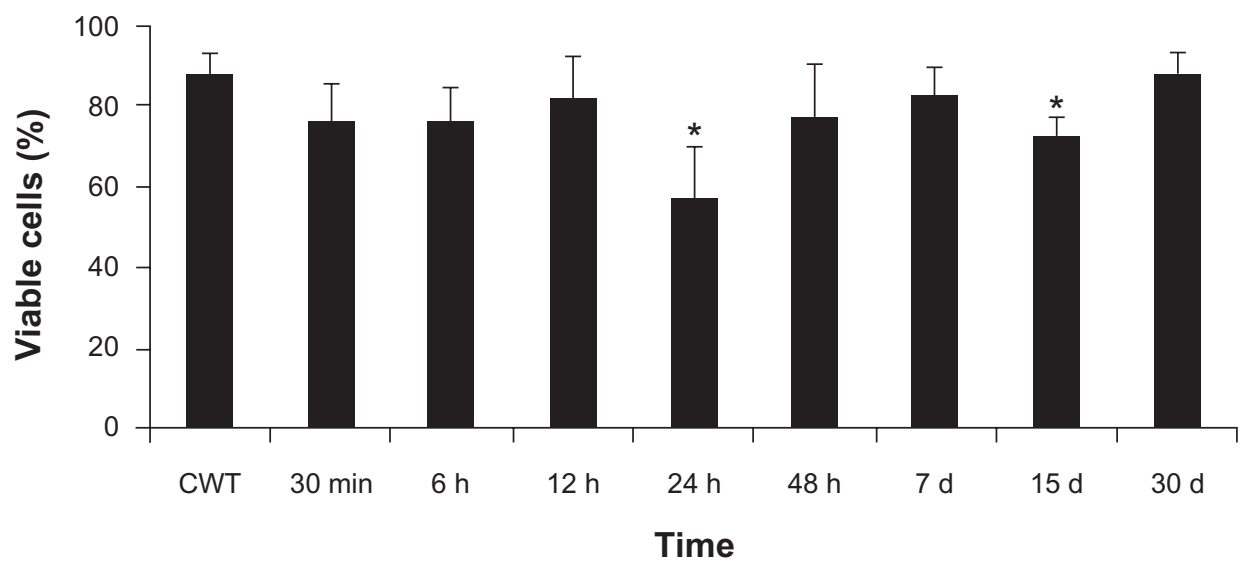

Figure 3 Effects of MAN administration on the index of viable peritoneal cells as a function of time.

Note: *Significant differences between CWT and MAN treatment by Tukey's post-hoc test: $P<0.05$.

Abbreviations: CWT, control group without treatment; MAN, magnetic albumin nanosphere.

administration of cyclophosphamide significantly increased this value to $36.50 \pm 5.39(P=0.000)$, as expected while using this positive control. Furthermore, no significant change was observed in the \% PCE after MAN administration (Table 1).

\section{Histological analysis}

Observation of MNP clustering was carried out while analyzing the histological slides of the brain. MNP clusters were visualized as brown or blue pigment after hematoxylin and eosin or Perls' staining, respectively. Clusters were first observed associated with erythrocytes (Figure 4A) of cerebral cortex capillaries, cerebellum, medulla, and also in areas of circumventricular organs (CVO). The highest concentration

Table I Effects of MAN administration on micronucleus frequency and polychromatic cell index

\begin{tabular}{llcll}
\hline Time & Group & MN-PCE & MN-NCE & \% PCE \\
\hline \multirow{4}{*}{$24 \mathrm{~h}$} & CWT & $3.67 \pm 1.03$ & $2.00 \pm 1.10$ & $0.55 \pm 0.06$ \\
& PC & $36.50 \pm 5.39 *$ & $3.50 \pm 1.38$ & $0.49 \pm 0.07$ \\
& FBS & $1.67 \pm 2.08$ & $3.33 \pm 2.31$ & $0.54 \pm 0.02$ \\
$48 \mathrm{~h}$ & MAN & $4.33 \pm 2.94$ & $3.83 \pm 2.14$ & $0.49 \pm 0.04$ \\
& FBS & $3.00 \pm 1.00$ & $4.00 \pm 0.00$ & $0.55 \pm 0.01$ \\
$7 \mathrm{~d}$ & MAN & $3.17 \pm 1.72$ & $4.50 \pm 2.67$ & $0.49 \pm 0.04$ \\
& FBS & $3.33 \pm 0.58$ & $1.33 \pm 2.31$ & $0.51 \pm 0.01$ \\
$15 \mathrm{~d}$ & MAN & $4.17 \pm 1.17$ & $2.50 \pm 0.84$ & $0.49 \pm 0.04$ \\
& FBS & $4.67 \pm 1.53$ & $1.00 \pm 1.00$ & $0.51 \pm 0.01$ \\
$30 \mathrm{~d}$ & MAN & $4.17 \pm 0.41$ & $2.17 \pm 0.75$ & $0.53 \pm 0.03$ \\
& FBS & $2.00 \pm 1.00$ & $2.00 \pm 1.00$ & $0.53 \pm 0.05$ \\
& MAN & $2.50 \pm 1.23$ & $1.50 \pm 1.05$ & $0.52 \pm 0.06$ \\
\hline
\end{tabular}

Note: *Significant difference between CWT and cyclophosphamide treatment (PC) by Tukey's post-hoc test, with $P<0.005$.

Abbreviations: CWT, control group without treatment; PC, positive control treated with cyclophosphamide; FBS, fetal bovine serum; MAN, magnetic albumin nanosphere; MN-PCE, micronucleated polychromatic erythrocytes; MN-NCE, micronucleated normochromatic erythrocytes; \% PCE, percentage of polychromatic erythrocytes. of MNP clusters associated with the erythrocytes was detected at 12 hours and 7 days after treatment. The analyses clearly showed that MNPs crossed the blood-brain barrier (BBB) as shown in Figure 4B, following distribution among both glial (Figure 4C) and choroid plexus cells. Additionally, MNPs were also internalized by neurons (Figure 4D), mainly in granule cells from the cerebellum. The highest content of MNP clusters in neurons was found 2 days after the MAN treatment, followed by a reduction in MNP content 30 days after MAN injection. Apparently the glial cells are of the cellular type that accumulated not only the highest content of MNPs but also kept the MNP clusters for the longest time period in our experiments.
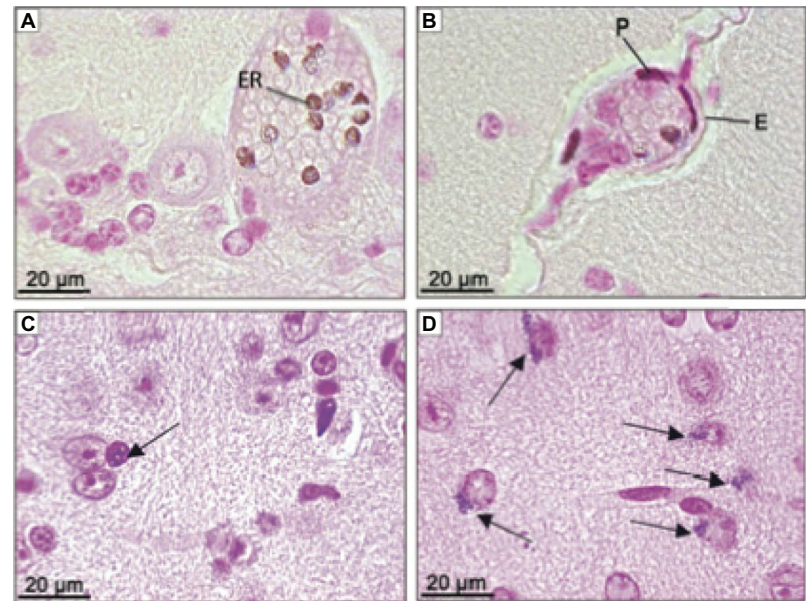

Figure 4 Photomicrographies of CNS showing ferric pigment in animals treated with MAN. (A) Erythrocytes in capillary from cerebellum 12 hours after treatment; (B) cells from BBB 12 hours after treatment; (C) glial cell (arrow) next to neurons from brain 24 hours after MAN treatment; (D) neurons from brain with nanoparticle clusters in one of the polar regions (arrow) 30 days after treatment. (A and $\mathbf{B}$ ) sections stained with hematoxylin and eosin; (C and D) sections stained by Perls' method. Abbreviations: $E R$, erythrocyte; $P$, pericyte; $E$, endothelial cell; MAN, magnetic albumin nanosphere; BBB, blood-brain barrier; CNS, central nervous system. 
MAN did not promote any histological alteration in the brain, liver, spleen, kidneys, heart, and pancreas, although MNP clusters were found in the central nervous system (CNS). The diluent FBS induced thickening of alveolar septa in control animals, and thus this effect was more intense in the lungs of MAN-treated animals (data not shown).

\section{Discussion}

In this study, MAN with $73.0 \pm 3.0 \mathrm{~nm}$ average diameter was considered biocompatible. This statement is based on the data showing no cytotoxic, genotoxic, or neurotoxic effects and only temporary alterations in both white cell populations (up to 7 days) and cell viability (up to 15 days after MAN application). Interestingly, MAN was able to cross the BBB safely, with no observable neurotoxicity, suggesting its potential use as MDDS, especially while targeting the CNS, an event only possible for a small number of drugs. ${ }^{21}$

It has been shown that the physical-chemical characteristics of MNPs, such as core-shell chemical composition, surface charge, and size, modulate MNP behavior in the biological environment. ${ }^{22-24}$ For instance, the surface coating of nano-sized material structures with non-opsonin serum proteins, such as albumin, represents a successful strategy to reduce recognition by the phagocytary mononuclear system cells, ${ }^{25,26}$ while presenting a negative charge in neutral $\mathrm{pH}$ and a hydrophilic surface. In this context, the temporary decrease in leukocytes in the animals' blood was possibly due to their migration to the peritoneum or any other targeted organ, as would be expected from a pro-inflammatory reaction to foreign particles. Late migration, especially of neutrophils, indicates that the phagocytary mononuclear system cells take longer to recognize the injected MAN. ${ }^{27-29}$ Thus, the combination of negatively charged surface and hydrophilicity embodied by MAN helps prevent major drawbacks in regard to leukocyte counts.

Differential count of peritoneal leukocytes was performed to check the possible occurrence of local inflammatory effects after the intraperitoneal administration of MAN. Significant differences were not observed in the most abundant peritoneal cell populations (macrophages and lymphocytes). However, some fluctuations in peritoneal cell populations related to the inflammatory response (neutrophils and eosinophils) ${ }^{19,30-32}$ were detected until 7 days, even after just one single injection of MAN, as observed previously. These fluctuations in peritoneal cell viability may be associated with the particulate state of the intraperitoneally administered MNPs and the consequent responses of neutrophil and eosinophil populations. After phagocytosis of a relatively high quantity of MAN, neutrophils die, releasing MNPs in the peritoneal cavity while reducing the viability index observed at 24 hours. Due to the particulate state of these released MNPs, more neutrophils are recruited to the peritoneum, increasing the peritoneal cell viability observed at 48 hours and 7 days. These fluctuations in neutrophil populations probably occur until the total clearance of MNPs from the peritoneum. Further, death of eosinophils from 8 to 12 days after migration to the peritoneum may explain, to a lesser degree, the decrease in cell viability at 15 days after the MAN treatment. The effects detected in cell viability were temporary and observed until 48 hours (neutrophil) or 7 days (eosinophil) after treatment, thus evidencing only a slight inflammatory process.

Strong evidence of MAN biocompatibility was obtained with the MN assay. Although micronuclei development may be associated with reactive oxygen species (ROS) induced by iron excess, ${ }^{23,33}$ in the present study, the MN assay showed no MAN effect on cell division or genotoxicity, differently from other samples based on magnetic nanoparticles. ${ }^{19,20,32}$ Also, the $\%$ PCE indicated that the MAN sample was not cytotoxic to the erythrocytic lineage in bone marrow.

MAN biodistribution and the possible induction of tissue alterations, such as inflammatory infiltration, thickening, and tissue necrosis were investigated through histological analyses. We hypothesize that individual MNPs, released from MAN due to bleaching while in the organism, were used to build clusters, thus suggesting MNP targets. MNP clusters were not visualized in the spleen, liver, pancreas, kidneys, or heart, differently from the data previously observed after administration of several magnetic fluid samples. ${ }^{11,19,20,31}$ It should be noted that the absence of histological alteration in the analyzed organs is another signature of MAN biocompatibility. On the other hand, MNP clusters were observed in the nervous tissue, suggesting the high specificity of this new albumin-based magnetic nanomaterial to the CNS. Interestingly, although MNP clusters were observed in the CNS, no histological alteration was detected in this organ. Transmission electron microscopy (TEM) investigations showed further evidence for this observation, ${ }^{34}$ suggesting that MAN reduced neurotoxicity.

The composition of iron and albumin seems to be important in understanding not only the observed MAN biocompatibility but also the cellular uptake mechanisms responsible for passage of MAN through the BBB, dispersion to the nervous tissue, and internalization by several CNS cellular types. 
The specificity of MAN to the CNS is in agreement with Bickel's report, ${ }^{35}$ which emphasized that albumin presents selectivity to brain tissue in rats, in comparison to other organs such as the liver, heart, and lungs. Other reports ${ }^{36,37}$ showed that polyethylene glycol-coated liposomes and polymeric nanoparticles, both based on bovine albumin, were able to cross the BBB. Data in the literature allow us to hypothesize that the ability of MAN to cross the BBB is partially due to the interaction of albumin with lipidic membranes. ${ }^{38}$ Transport could be mediated by specific receptors, such as gp60, which facilitate transcytosis through BBB endothelial cells by a tyrosine-kinase dependent pathway. ${ }^{39}$

Furthermore, changes in cell membrane permeability, induced by the presence of $\mathrm{Fe}^{3+}$ on the structure and function of the phospholipid bilayer, ${ }^{40}$ may help shed light on the ability of MAN to cross the BBB. The region of $\mathrm{CVO},{ }^{41}$ where the capillaries from $\mathrm{BBB}$ are more permeable to solutes, could also influence interactions between MAN and the CNS. When MAN reaches the CNS the initial targeted regions were very close to lateral ventricles and the CVO.

A number of reports showed that MNPs can enter the brain and cause tissue injury. ${ }^{42}$ Previous studies showed that high concentrations of anionic or cationic nanoparticles (NPs) disrupted the BBB, whereas neutral NPs and low concentrations of anionic NPs had no effect on BBB integrity. ${ }^{43}$ Further, interactions of ferric ions with phospholipids of bilayers may lead to the disruption of the structure of cellular membrane ${ }^{44}$ and decrease the fluidity of neuronal membranes. ${ }^{45}$ The structural changes in membranes may lead to higher internalization of iron through changes in permeability. Thus, iron could interact with structures of neuronal cytoskeletons, acting on the axonal transport system and on synapses. In our study, it was found that glial cells were the cellular type that accumulated the highest content of MNPs and kept them for the longest period. It is known that glial cells ${ }^{46}$ accumulate a high concentration of iron when there is an injury, but are rarely seen accumulating iron in normal conditions. ${ }^{47}$ Nevertheless, no alterations were detected in glial cells, even under TEM analysis, ${ }^{34}$ thus suggesting that the MAN surface chemical-physical characteristics protect CNS structures from injuries and that the released iron is not enough to induce reactive oxygen species generation. ${ }^{48}$

The finding of MAN biocompatibility is an important milestone in nanotoxicity research as it can foster future studies related to biomedical applications of MAN-related materials. The observed capacity of MAN to cross the BBB with reduced neurotoxicity is extremely promising for targeting diseases related to the CNS, such as Parkinson's disease, Alzheimer's disease, and multiple sclerosis. ${ }^{7,49}$ In addition, the authors firmly believe that MAN represents a promising material vehicle to selectively destroy cancer cells, including brain tumors, mainly because those cells overexpress a number of receptors to albumin. ${ }^{12,17}$ MAN can be loaded with different materials, with the purpose of either diagnosis or therapy, ${ }^{6,15}$ since albumin presents a number of reactive amino and carboxylic groups. The potential of MAN in tumor treatment using magnetohyperthermia or carrying sensitizers for photodynamic therapy has been recently shown by in vitro ${ }^{15,40}$ and in vivo ${ }^{6}$ investigations.

\section{Conclusion}

It is concluded that the designed and investigated magnetic albumin nanosphere (MAN) represents a biocompatible and safe material. This affirmation is mainly based on the data presenting absolutely no cytotoxic and genotoxic effects, nor any morphological alterations while tested using an animal model. MAN induced some slight and transitory alterations in other nanotoxicity tests that do not interfere in its biocompatibility status. Actually, data revealed the importance of investigating nanomaterials for a longer time window because some biological responses may be reversed after a relatively long period of time, as observed in the viability tests. Finally, the presence of MNP clusters in the central nervous system evidences the high specificity of MAN in targeting the brain, suggesting its use as a new MDDS that is able to cross the $\mathrm{BBB}$, with reduced neurotoxicity, and thus representing a promising material platform for therapy of brain-related diseases.

\section{Acknowledgments}

This research was supported by Brazilian agencies MCT/ CNPq, CAPES-Rede CON-NANO, FAP-DF, FINATEC, INCT-Nanobiotecnologia, CNANO, and DPP-UnB.

\section{Disclosure}

The authors report no conflicts of interest in this work.

\section{References}

1. Torchilin VP. Targeted pharmaceutical nanocarriers for cancer therapy and imaging. AAPS J. 2007;9(2):E128-E147.

2. Dilnawaz F, Singh A, Mohanty C, Sahoo SK. Dual drug loaded superparamagnetic iron oxide nanoparticles for targeted cancer therapy. Biomaterials. 2010;31(13):3694-3706. 
3. De Jong WH, Borm PJ. Drug delivery and nanoparticles:applications and hazards. Int J Nanomedicine. 2008;3(2):133-149.

4. Chomoucka J, Drbohlavova J, Huska D, Adam V, Kizek R, Hubalek J. Magnetic nanoparticles and targeted drug delivering. Pharmacol Res. 2010;62(2):144-149.

5. Guedes MHA, Sadeghiani N, Peixoto DLG, et al. Effects of AC magnetic field and carboxymethyldextran-coated magnetite nanoparticles on mice peritoneal cells. Jof Magn Magn Mater. 2005;293(1): 283-286.

6. Portilho FA, Estevanato LLC, Miranda-Vilela AL, et al. Investigation of a magnetohyperthermia system efficacy. J App Phys. 2010;109(7) B307-B309.

7. Murthy SK. Nanoparticles in modern medicine: state of the art and future challenges. Int J Nanomedicine. 2007;2(2):129-141.

8. McBain SC, Yiu HH, Dobson J. Magnetic nanoparticles for gene and drug delivery. Int J Nanomedicine. 2008;3(2):169-180.

9. Gupta AK, Gupta M. Synthesis and surface engineering of iron oxide nanoparticles for biomedical applications. Biomaterials. 2005;26(18): 3995-4021.

10. Mornet S, Vasseur S, Grasset F, et al. Magnetic nanoparticle design for medical applications. Progr Solid State Chem. 2006;34(2-4): $237-247$.

11. Lacava LM, Garcia VAP, Kückelhaus S, et al. Long-term retention of dextran-coated magnetite nanoparticles in the liver and spleen. J Magn Magn Mater. 2004;272-276(Pt 3):2434-2435.

12. Kratz F. Albumin as a drug carrier: Design of prodrugs, drug conjugates and nanoparticles. J Control Release. 2008;132(3):171-183.

13. Li J-m, Chen W, Wang H, et al. Preparation of albumin nanospheres loaded with gemcitabine and their cytotoxicity against BXPC-3 cells in vitro. Acta Pharmacol Sin. 2009;30(9):1337-1343.

14. Miele E, Spinelli GP, Miele E, Tomao F, Tomao S. Albumin-bound formulation of paclitaxel (Abraxane ${ }^{\circledR} \mathrm{ABI}-007$ ) in the treatment of breast cancer. Int J Nanomedicine. 2009;4:99-105.

15. Simioni AR, Rodrigues MMA, Primo FL, Morais PC, Tedesco AC. Effect of diode-laser and AC magnetic field of bovine serum albumin nanospheres loaded with phthalocyanine and magnetic particles. J Nanosci Nanotechnol. 2011;11(4):3604-3608.

16. Figueiredo LC, Lacava BM, Skeff Neto K, Pelegrini F, Morais PC. Magnetic resonance study of maghemite-based magnetic fluid. J Magn Magn Mater. 2008;320(14):e347-e350.

17. Simioni AR, Martins OP, Lacava ZG, et al. Cell toxicity studies of albumin-based nanosized magnetic beads. J Nanosci Nanotechnol. 2006;6(8):2413-2415.

18. Morais PC, Garg VK, Oliveira AC, et al. Synthesis and characterization of size-controlled cobalt-ferrite-based ionic ferrofluids. J Magn Magn Mater. 2001;225(1-2):37-40.

19. Freitas MLL, Silva LP, Azevedo RB, et al. A double-coated magnetitebased magnetic fluid evaluation by cytometry and genetic tests. J Magn Magn Mater. 2002;252:396-398.

20. Kückelhaus S, Reis SC, Carneiro MF, et al. In vivo investigation of cobalt ferrite-based magnetic fluid and magnetoliposomes using morphological tests. J Magn Magn Mater. 2004;272-276(Pt 3): 2402-2403.

21. Laquintana V, Trapani A, Denora N, Wang F, Gallo JM, Trapani G. New strategies to deliver anticancer drugs to brain tumors. Expert Opin Drug Deliv. 2009;6(10):1017-1032.

22. Dobrovolskaia MA, McNeil SE. Immunological properties of engineered nanomaterials. Nat Nanotechnol. 2007;2(8):469-478.

23. Jain TK, Reddy MK, Morales MA, Leslie-Pelecky DL, Labhasetwar V. Biodistribution, clearance, and biocompatibility of iron oxide magnetic nanoparticles in rats. Mol Pharm. 2008;5(2):316-327.

24. Lacava ZGM, Tedesco AC, Azevedo RB, Morais PC. The use of magnetic nanoparticles in drug-delivery systems: the interaction of nanoparticles with cells. In: Kumar MNV, editor. Handbook of Particulate Drug Delivery. California: American Scientific Publishers; 2008:223-233.
25. Ogawara K, Furumoto K, Nagayama S, et al. Pre-coating with serum albumin reduces receptor-mediated hepatic disposition of polystyrene nanosphere: implications for rational design of nanoparticles. J Control Release. 2004;100(3):451-455.

26. Leroux JC, De Jaeghere F, Anner B, Doelker E, Gurny R. An investigation on the role of plasma and serum opsonins on the internalization of biodegradable poly(D,L-lactic acid) nanoparticles by human monocytes. Life Sci. 1995;57(7):695-703.

27. Chellat F, Merhi Y, Moreau A, Yahia L. Therapeutic potential of nanoparticulate systems for macrophage targeting. Biomaterials. 2005;26(35):7260-7275.

28. Owens DE, Peppas NA. Opsonization, biodistribution, and pharmacokinetics of polymeric nanoparticles. Int J Pharm. 2006;307(1):93-102.

29. Vonarbourg A, Passirani C, Saulnier P, Benoit JP. Parameters influencing the stealthiness of colloidal drug delivery systems. Biomaterials. 2006;27(24):4356-4373.

30. Lacava ZGM, Azevedo RB, Lacava LM, et al. Toxic effects of ionic magnetic fluids in mice. J Magn Magn Mater. 1999;194(1-3):90-95.

31. Garcia MP, Parca RM, Chaves SB, et al. Morphological analysis of mouse lungs after treatment with magnetite-based magnetic fluid stabilized with DMSA. J Magn Magn Mater. 2005;293(1):277-282.

32. Sadeghiani N, Barbosa LS, Guedes MHA, et al. Magnetic resonance of polyaspartic acid-coated magnetite nanoparticles administered in mice. IEEE Trans Magn. 2005;41(10):4108-4110.

33. Premkumar K, Bowlus CL. Ascorbic acid reduces the frequency of iron induced micronuclei in bone marrow cells of mice. Mutat Res. 2003;542(1-2):99-103.

34. Cintra DOS, Estevanato LLC, Simioni AR, et al. Successful strategy for targeting the central nervous system using magnetic albumin nanospheres. J Biomed Nanotechnol. In press 2011.

35. Bickel U, Yoshikawa T, Pardridge WM. Delivery of peptides and proteins through the blood-brain barrier. Adv Drug Deliv Rev. 2001;46(1-3):247-279.

36. Thole M, Nobmann S, Huwyler J, Bartmann A, Fricker G. Uptake of cationzied albumin coupled liposomes by cultured porcine brain microvessel endothelial cells and intact brain capillaries. J Drug Target. 2002;10(4):337-344

37. Lu W, Tan YZ, Hu KL, Jiang XG. Cationic albumin conjugated pegylated nanoparticle with its transcytosis ability and little toxicity against blood-brain barrier. Int J Pharm. 2005;295(1-2):247-260.

38. Yang H. Nanoparticle-Mediated Brain-Specific Drug Delivery, Imaging, and Diagnosis. Pharm Res. 2010;27(9):1759-1771.

39. Vogel SM, Minshall RD, Pilipovic M, Tiruppathi C, Malik AB. Albumin uptake and transcytosis in endothelial cells in vivo induced by albuminbinding protein. Am J Physiol Lung Cell Mol Physiol. 2001;281(6): L1512-L1522.

40. Simioni AR, Primo FL, Rodrigues MMA, Lacava ZGM, Morais PC, Tedesco AC. Preparation, characterization and in vitro toxicity test of magnetic nanoparticle-beads drug delivery system to hyperthermia of biological tissues. IEEE Trans Magn. 2007;43(6):2459-2461.

41. Misra A, Ganesh S, Shahiwala A, Shah SP. Drug delivery to the central nervous system: a review. J Pharm Pharm Sci. 2003;6(2):252-273.

42. Hu Y-L, Gao J-Q. Potential neurotoxicity of nanoparticles. Int J Pharm. 2010;394(1-2):115-121.

43. Lockman PR, Koziara J, Roder KE, et al. In vivo and in vitro assessment of baseline blood-brain barrier parameters in the presence of novel nanoparticles. Pharm Res. 2003;20(5):705-713.

44. Suwalsky M, Martínez F, Cárdenas H, Grzyb J, Strzalka K. Iron affects the structure of cell membrane molecular models. Chem Phys Lipids. 2005;134(1):69-77.

45. Ohba S, Hiramatsu M, Edamatsu R, Mori I, Mori A. Metal ions affect neuronal membrane fluidity of rat cerebral cortex. Neurochem Res. 1994;19(3):237-241.

46. Banati RB. Neuropathological imaging: in vivo detection of glial activation as a measure of disease and adaptive change in the brain. $\mathrm{Br} \mathrm{Med}$ Bull. 2003;65(1):121-131. 
47. Sastry S, Arendash GW. Time-dependent changes in iron levels and associated neuronal loss within the substantia nigra following lesions within the neostriatum/globus pallidus complex. Neuroscience. 1995;67(3):649-666.

48. Naqvi S, Samim M, Abdin MZ, et al. Concentration-dependent toxicity of iron oxide nanoparticles mediated by increased oxidative stress. Int J Nanomedicine. 2010;5:983-989.
49. Van Kasteren SI, Campbell SJ, Serres S, Anthony DC, Sibson NR, Davis BG. Glyconanoparticles allow pre-symptomatic in vivo imaging of brain disease. Proc Natl Acad Sci U S A. 2009;106(1):18-23.

International Journal of Nanomedicine

\section{Publish your work in this journal}

The International Journal of Nanomedicine is an international, peerreviewed journal focusing on the application of nanotechnology in diagnostics, therapeutics, and drug delivery systems throughout the biomedical field. This journal is indexed on PubMed Central, MedLine, CAS, SciSearch $\AA$, Current Contents ${ } /$ Clinical Medicine,
Journal Citation Reports/Science Edition, EMBase, Scopus and the Elsevier Bibliographic databases. The manuscript management system is completely online and includes a very quick and fair peer-review system, which is all easy to use. Visit http://www.dovepress.com/ testimonials.php to read real quotes from published authors.

Submit your manuscript here: http://www.dovepress.com/international-journal-of-nanomedicine-journal 